\title{
Load Balancing Using Multiple Node Disjoint Paths
}

\author{
Ahmad Haboush ${ }^{1}$, Mohammad Al Nabhan ${ }^{1}$, Motassem Al-Tarazi ${ }^{1} \&$ Mohammad Al-Rawajbeh ${ }^{2}$ \\ ${ }^{1}$ Computer Science and Network Department, Jerash University, Jordan \\ ${ }^{2}$ Management Information Systems Department, Isra University, Jordan \\ Correspondence: Ahmad Haboush, Chiarman of Computer Science and Network Department, Jerash University, \\ Jordan. E-mail: ahmad_ram2001@yahoo.com
}

Received: February 27, 2012 Accepted: March 15, 2012 Online Published: May 1, 2012

doi:10.5539/cis.v5n3p83

URL: http://dx.doi.org/10.5539/cis.v5n3p83

\begin{abstract}
This paper discusses the multiple node disjoint paths protocol (MNDP) for mobile ad hoc networks. A mobile ad hoc network is a collection of mobile nodes that cooperate without networking infrastructure so as to form a temporary network that meets some immediate needs. The MNDP protocol detects multiple paths and distributes transmitted packets over these paths. Such distribution reduces congestion and packet end-to-end delay, and increase the delivery ratio. The MNDP protocol detects multiple paths, assigns them a priority values based on the routes hop count and uses the shortest routes among them more frequently. The simulation results show that the proposed protocol has achieved an enhancement on packet delivery ratio, up to $16 \%$, as compared to the $\mathrm{Ad}$ Hoc On-demand Distance Vector routing protocol (AODV) protocol. Finally, the results are obtained based by the GloMoSim 2.03 simulations.
\end{abstract}

Keywords: load balancing, multiple routes, ad hoc networks, congestion, node disjoint

\section{Introduction}

Mobile Ad hoc networks (MANETs) are groups of infrastructureless nodes that are typically created dynamically within a rapid topology. MANETs have many restrictions that make it complicated, such as: its nodes are battery powered, resources are limited, the topology of the network can change frequently, and the area that can be covered by a node's transmission is limited. Therefore, nodes need to act both as router and host (MANET, 2008).

One of the most important issues to be discussed in MANETs is routing. Routing is the process of determining a route for transferring messages and packets from a source node to a destination node (Perkins \& Royer, 1999). A number of routing protocols have been proposed to cope with ad hoc networks characteristics. These protocols can be categorized as proactive and reactive protocols or single-path and multi-path protocols.

Our study is based on the Ad hoc on demand distance victor (AODV) routing protocol which chooses a single shortest path in order to transmit data packets between a source and distention pair. The main disadvantage of using such route is the congestion problem. Congestion occurs when the number of packets being transmitted through a node begins to approach the packet-handling capacity of the node. The MNDP is used to prevent the network of having congestion by dispersing the transmitted packets over multiple routes.

The main objective of this paper is to increase the packet delivery ratio by reducing congestion. To achieve this objective, we developed a multiple node-disjoint paths protocol (MNDP), which is based on multi-path routing and load distribution. The idea is to statistically distribute the load onto multiple routes so as to reduce congestion.

The rest of the paper is organized as follows: In section two, we review the Multi-Path Routing Algorithms and Load Balancing. Related work is presented in section three. Section Four explores the development of MNDP protocol. In section Five, we evaluate the performance of MNDP. Section six concludes this work and provides suggestions for future work.

\section{Multi-path Routing Algorithms}

A network is said to have multiple paths when there exists two or more routes between the same source-destination pair. The purpose of having multiple paths is either enhancing the reliability of the data transmission (fault-tolerance), or allowing load balancing. For fault-tolerance, the source sends the same message via a number of routes at the same time in order to increase delivery reliability. If a path has failed, the 
message is likely to be received via other paths (Ye et al., 2003). Routes discovered by multiple-path protocols can be classified into node disjoint paths and link disjoint paths.

In Node-disjoint paths, each node in the route can participate only in one path, except the source and destination nodes (Tsai \& Moors, 2006). Any node failure will result in a maximum of one broken path for a given source, as shown in Figure 1.

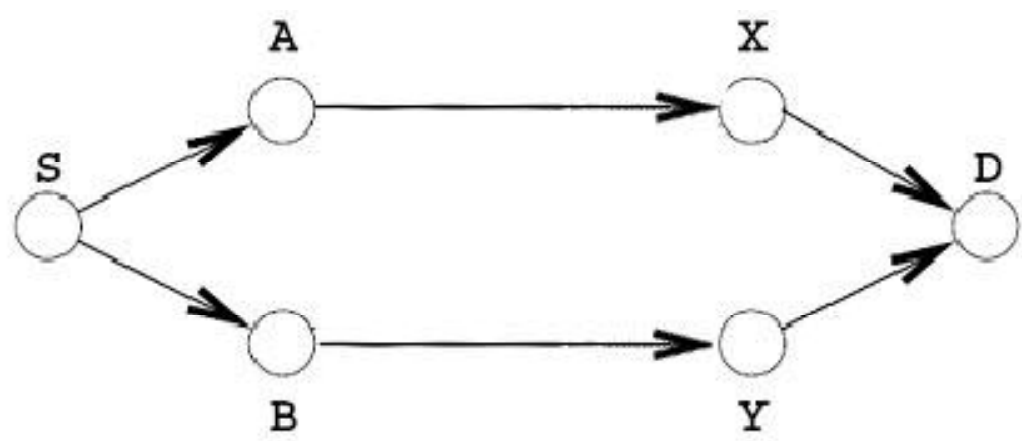

Figure 1. Two node-disjoint routes

Several protocols have been proposed for multiple-path discovery. They include the source routing protocol Split Multipath Routing (SMR) (Lee \& Gerla, 2001), the Ad hoc On-demand Multipath Distance Vector (AOMDV) routing (Marina \& Das, 2001), and the Ad hoc On-demand Distance Vector Multipath (AODVM) protocol (Ye et al., 2003).

Load-balancing is a technique that is used to distribute the load to other nodes in order to decrease the congestion at intermediate nodes, and improve the packet delivery ratio and packet delay. Load-balancing techniques can be used in both single-path and multiple-path protocols. It is obvious that efficient load-balancing can be achieved when combined with multiple paths in high density networks (i.e., networks with a large number of paths).However, when the network density is low (number of paths discovered is low), multiple path protocols may not balance the load better than single-path protocols (Ganjali \& Keshavarzian, 2004).

\section{Related Work}

Souihli et al. (Souihli et al., 2009) show that intermediate nodes located on the shortest paths have the highest load in the network. Their idea depends on pushing the load from shortest paths that goes through the network center onto other routes at the edges of the network. Souihli et al. propose using a degree of centrality that determines the closeness of a node to the center of the network. A high degree value gives an indication that a node might be located in the central area of the network, and a low value gives an indication that the node might be at the edge of the network (Souihli et al., 2009).

Lee and Gerla (2001) proposed the Dynamic Load-Aware Routing (DLAR) protocol, which uses the current load as selection metric. In the route discovery process, the intermediate nodes are not allowed to send a route reply in order to have up-to-date network load information. The current load is calculated in three ways; summing the current loads of intermediate nodes and select the route that has the minimum cumulative load, or by selecting a route that has the minimum average load or by Defining a threshold for the number of packets buffered in the intermediate nodes and select a route that has the minimum number of hops that exceed this threshold (Lee \& Gerla, 2001).

Ye et al. (Ye et al., 2004) proposed a Distributed Congestion-Aware Routing (DCAR) protocol for TCP connections. They proposed a congestion-aware mechanism where each node periodically sends a HELLO message to its 1-hop neighbors. The message contains the node congestion weight. Thus, each node has full information about the network, which allows having several synchronized TCP paths (Ye et al., 2004).

Hogan et al. (Hogan et al., 2004) proposed a localized congestion control solution that handles congestion by re-routing packets onto non-congested routes. If this mechanism does not reduce congestion, sources are prevented from adding any more packets to the network via routes that they know have congested nodes (Hogan et al., 2004). 
Lu et al. (Lu et al., 2003) proposed the Congestion-Aware Distance Vector (CADV) routing protocol, which is based on the integration of a proactive routing protocol and a congestion control mechanism. Each node calculates its expected delay based on the mean of delay for all data packets that it sent during the near past. The routing decision is made depending on the number of hops to the destination as well as the expected delay (Lu et al., 2003).

Zhou and Hassanein (2001) proposed the Load-Balanced Ad hoc Routing (LBAR) protocol. They defined a new routing metric called the degree of nodal activity, which is the number of active paths that a node participates in. This is an indirect measurement of node traffic. In order to avoid congested routes, the destination selects the minimum cost paths that have the minimum degree of nodal activity.

Sambasivam, Murthy and M. Belding-Royer (Ye et al., 2004) increase the data packets delivery ratio by sending data packets over high signal nodes. They detect multiple paths during route discovery process. They use the unicast periodic update packets to find multiple paths. The algorithm sends data packets over highest signal strength which improves the data packet delivery ratio. But the key drawback of this algorithm is that these paths might not be the shortest paths which may increase the end-to-end delay (Ye et al., 2004).

Ahn et al. (Tran \& Raghavendra, 2006) use standard AODV to detect a primary route. When this route is found, the source node starts sending data packets over this route while another route discovery process is initiated to have another backup route. The primary route and the backup route are node-disjoint routes. When the primary route fails, the source node starts sending data packets over the backup route (Tran \& Raghavendra, 2006).

\section{The Multiple Node Disjoint Paths Protocol (MNDP)}

Most MANET routing protocols do not include congestion control mechanisms. They use the shortest path, although the shortest path may suffer from congestion problems. To reduce the probability of having congested routes, we transmit data packets over a number of routes based on assigned priorities. In our proposal, the priority of a route depends on the number of routes used, denoted as Max_Routes, and their hop counts.

Max_Routes is the maximum number of routes over which data packets can be forwarded. Now we will describe our idea.

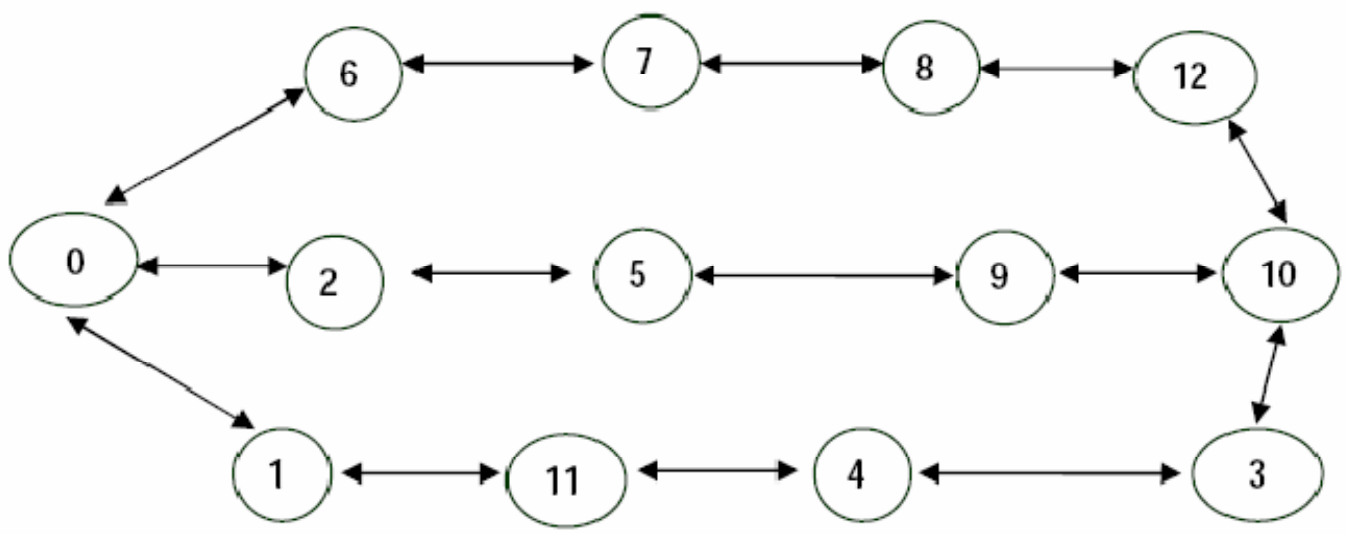

Figure 2. A group of nodes represents different active routes

In Figure 2, the network represents a group of mobile nodes, assuming that Max_Routes $=2$. If the source node 0 wants to send data packets to the destination node 3 , it will initiate a route discovery process. Assuming that the resulting routes are $\{0-2-5-9-10-3\}$ with a hop count of 5 , and $\{0-1-11-4-3\}$ with a hop count of 4 .

The source node will sort these routes based on the hop count value, and assign a route priority to each route. The shortest one will have a route priority equal to two, and the next route's priority will be set to one. If two routes have the same length, the older one will be placed before the newer route in the sorted list of routes. In general, if we have $n$ routes sorted in the non-decreasing order of their hop counts, they will be respectively assigned the priority values $n, n-1, n-2, \ldots \ldots, 2,1$. Assuming the transmitted load $\mathrm{L}$, the shorter route will carry $66.6 \%$ of the load, while the other route will hold $33.4 \%$ of the load. This way, we aim to avoid congested areas by distributing data packets over multiple routes, giving priority to transmitting over routes that are relatively short. Simulation results have shown that this choice of priorities values gives good performance. 
Route priorities are computed as the source node receives RREP messages. A source node selects a number of shortest paths (based on Max_Routes), and assigns a route priority value to each selected route. In this research, we have experimented with $\bar{M}$ ax_Routes values of 2, 3, 4, 5 and 6 .

\section{Simulation Results}

We have evaluated the performance of AOMDV with respect to AODV using GloMoSim 2.03 simulations under a wide range of mobility and traffic scenarios (Bajaj et al., 2009). Table 1 shows the simulator parameters which used for the several scenarios presented in the next section. To comprehensively measure the performance of our protocols, we used various pause times: 0, 100, 200 and 300 seconds. In addition, we used traffic loads of 1, 2, 4 and 8 packets per second, repeated for 5, 10 and 15 sources.

Table 1. Environment parameters (no mention of this table in the body of paper)

\begin{tabular}{ll}
\hline Parameter & Value \\
\hline Number of nodes & 30 \\
Medium Bandwidth & $2 \mathrm{Mb} / \mathrm{s}$ \\
Simulation time & 300 second \\
Application Model & $(\mathrm{CBR})$ \\
Network Dimensions & $1000 * 1000$ \\
Node Mobility & Random Waypoint \\
\hline
\end{tabular}

There are many performance metrics that can be used in order to assess the routing protocols. The Data delivery ratio and End-to-End delay are used to evaluate the enhancement of MNDP protocol as compared to the AODV protocol.

\subsection{Packet Delivery Ratio}

In Figure 3, when the traffic load is low (1 packet per sec), AODV outperforms our proposed algorithms. Our proposed algorithms send packets over a number of routes that may be long, which increases the probability that a data packet is dropped. In Figure 4 and Figure 5, when the traffic load is high (8 packets per sec), our proposed algorithms out perform AODV. Congestion might happen in AODV and data packets might be dropped which decreases the packet delivery ratio. Our proposed algorithms, distribute the load over multiple routes, so the probability of a congestion to occur will be small.

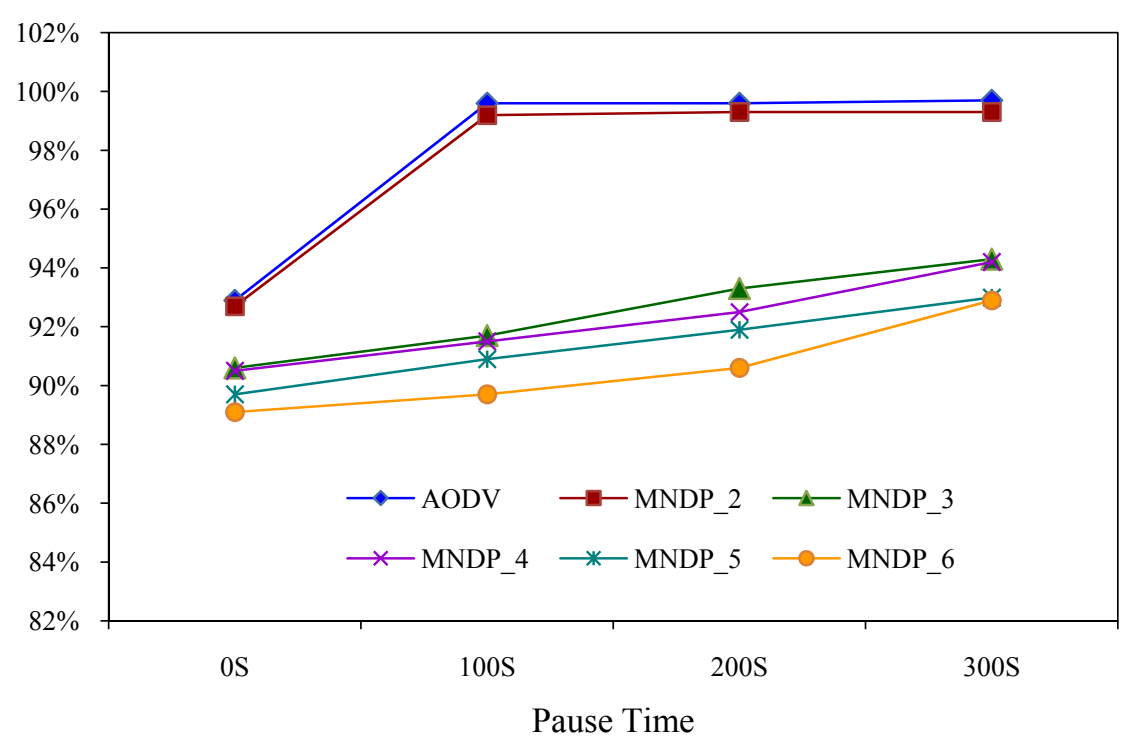

Figure 3. Delivery ratio of MNDP and AODV for 5 sources, sending 1 packet per sec 


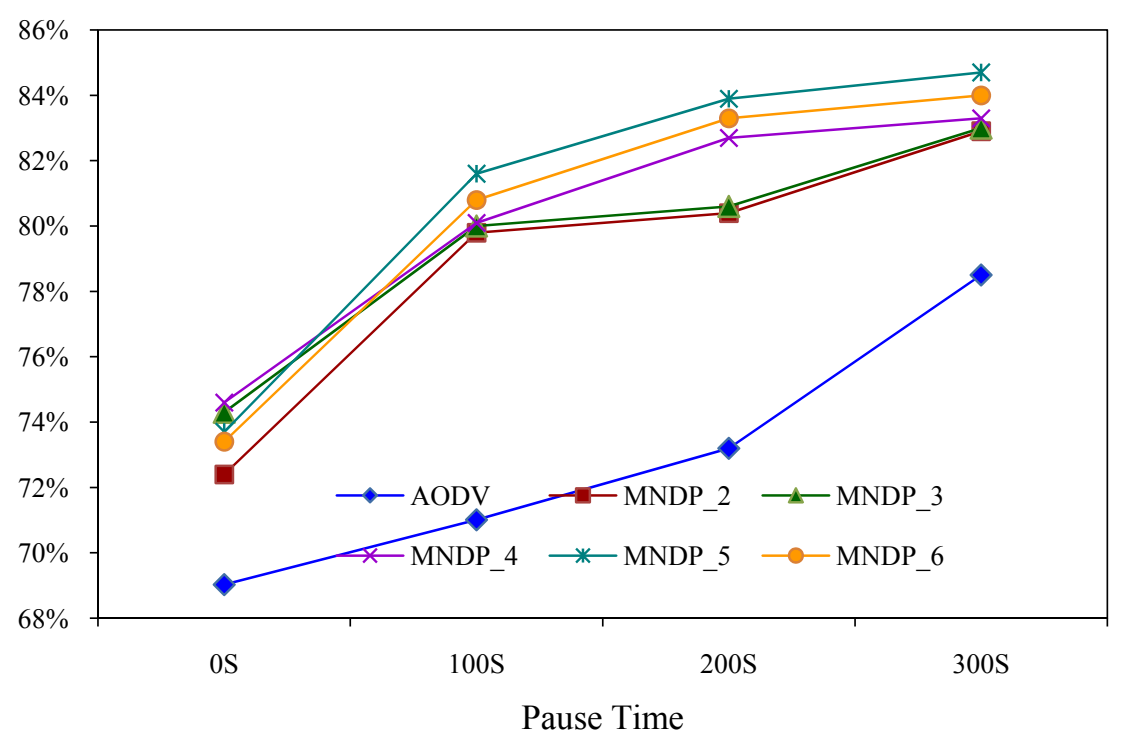

Figure 4. Delivery ratio of MNDP and AODV for 10 sources, sending 8 packets per sec

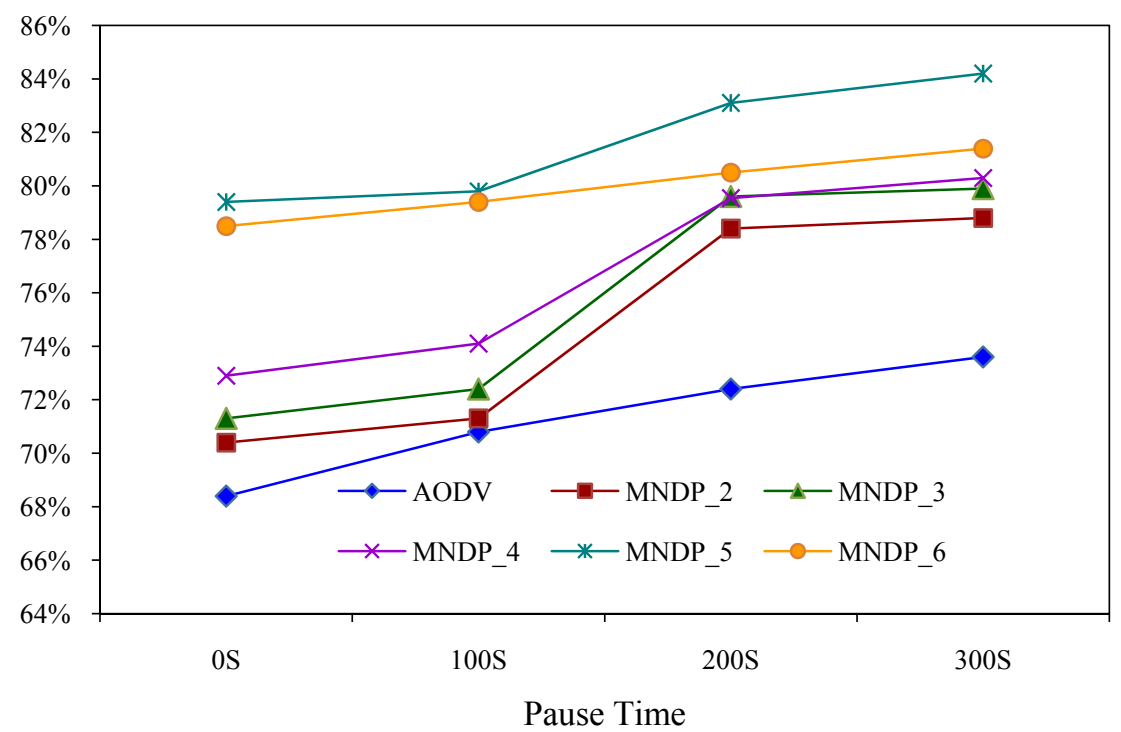

Figure 5. Delivery ratio of MNDP and AODV for 15 sources, sending 8 packets per sec

\subsection{Average End-to-end Delay}

In Figure 6, AODV is superb, our proposed protocols send data packets over a number of routes that are relatively long even if the traffic load is low (1 packet per sec), which increases the end-to-end delay. While in Figure 7 and Figure 8, when the traffic load is high ( 8 packets per sec), our proposed algorithms out perform AODV, because a congestion might happen and data packets might be dropped. These dropped packets eventually will be retransmitted again which increases the average end-to-end delay. 


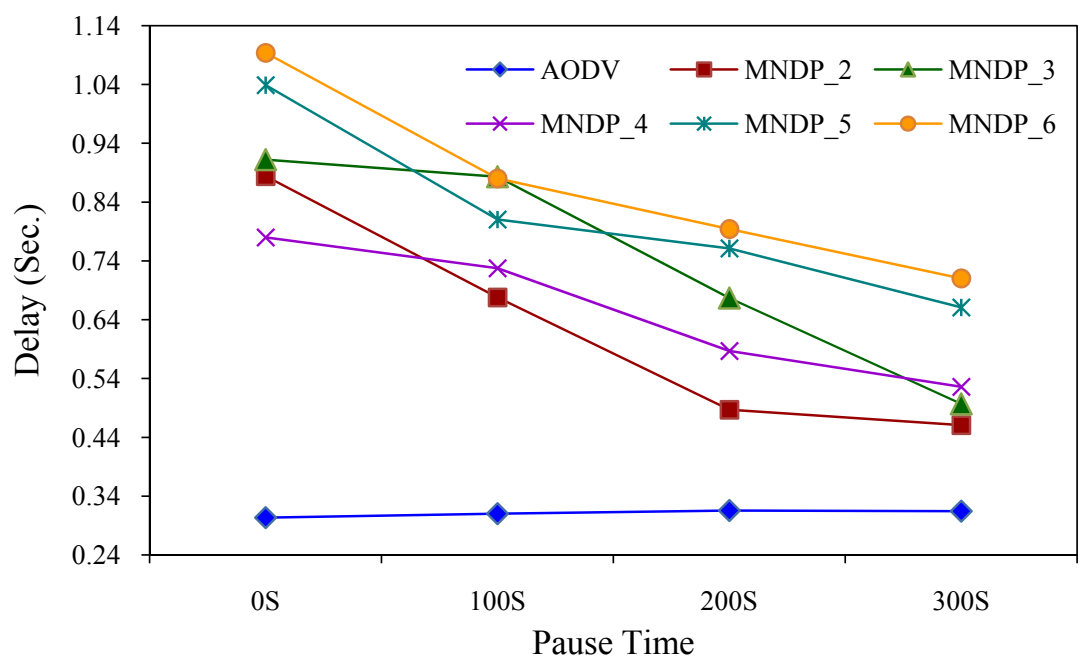

Figure 6. Average end-to-end delay of MNDP and AODV for 10 sources, sending 1 packet per sec

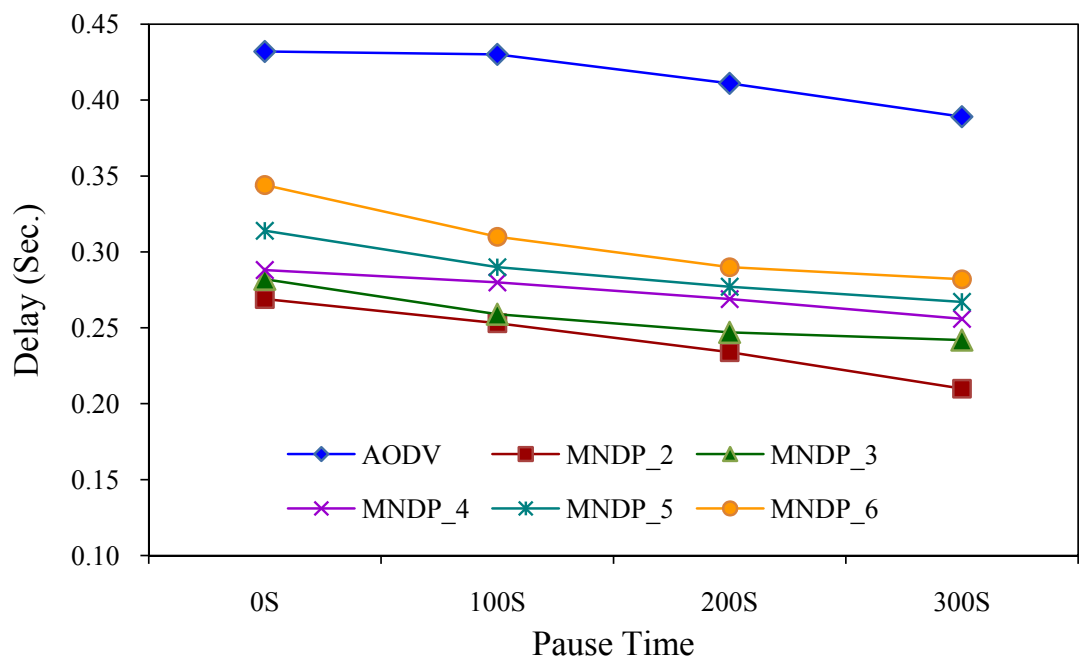

Figure 7. Average end-to-end delay of MNDP and AODV for 5 sources, sending 8 packets per sec

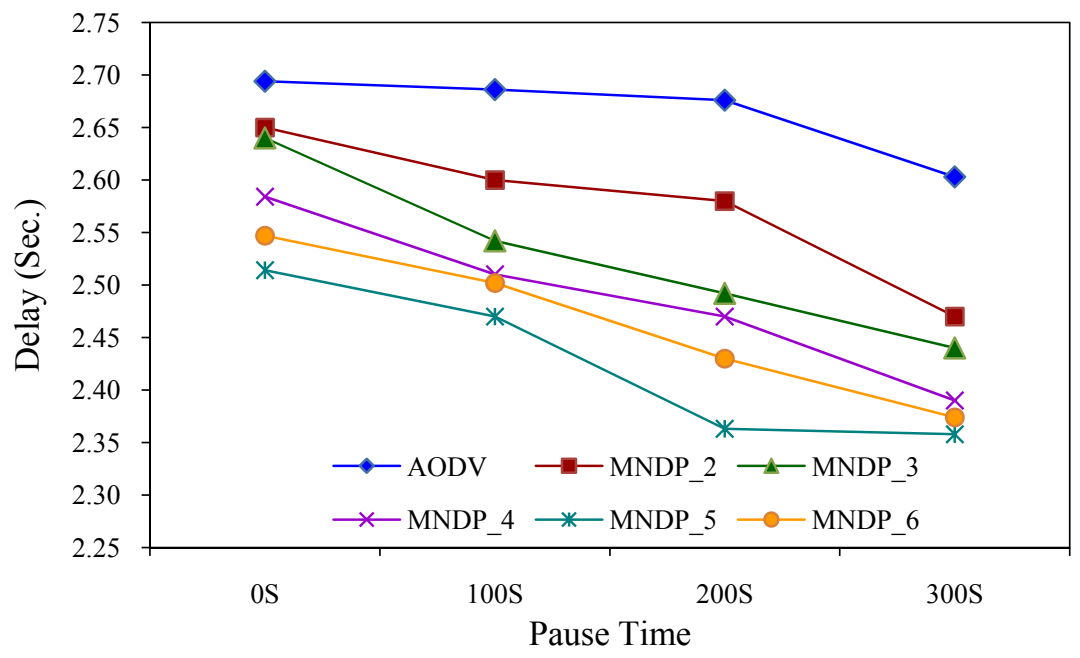

Figure 8. Average end-to-end delay of MNDP and AODV for 15 sources, sending 8 packets per sec 


\section{Conclusion}

In this paper, we have proposed load balancing protocols that detect multiple routes, assign a priority value to each route and send data packets over these routes, depending on the priority values.

We have studied the performance of MNDP relative to AODV under a wide range of mobility and traffic scenarios. We observe that MNDP offers a significant reduction in end to end delay and an increment in data packet delivery ratio.

The proposed schemes in this thesis use the hop count as selection metric. However, the shortest routes may be congested; therefore, schemes that consider the current congestion level as a selection metric should be investigated in the future. Also, alternative routes may be selected based on node mobility. In this case, preference should be given to choosing slow moving nodes.

\section{References}

Bajaj, L., Takai, M., Ahuja, R., Tang, K., Bagrodia, R., \& GloMoSim, A. (1999). A scalable network simulation environment technical report. UCLA Computer Science Department.

Ganjali, Y., \& Keshavarzian, A. (2004). Load balancing in Ad Hoc Networks: Single-path Routing vs. Multi-path Routing, INFOCOM 2004, Twenty-third Annual Joint Conference of the IEEE Computer and Communications Societies, vol 2, pp. 1120-1125.

Hogan, B., Barry, M., \& McGrath, S. (2004). Congestion avoidance in source routed Ad Hoc Networks. in proceedings of the 13th IST Mobile and Wireless Communications Summit, June.

IETF Mobile Ad Hoc Networks (MANET) Working Group Charter. (Online] [Accessed 2008 October]. Available: http://www.ietf.org/html.charters/manet-charter.html

Lee, S., \& Gerla, M. (2001). Dynamic load-aware routing in Ad hoc Networks. IEEE international, IEEE ICC, June, pp. 3206-10.

Lee, S., \& Gerla, M. (2001). Split multipath routing with maximally disjoint paths in Ad hoc Networks, in Proceedings of the IEEE International Conference on Communication (ICC2001), pp. 3201-3205.

Lu, Y., Wang, W., Zhong, Y., \& Bhargava, B. (2003).Study of distance vector routing protocols for mobile Ad Hoc Networks, in proceedings of the IEEE International Conference on Pervasive Computing and Communications (PerCom), pp. 187-194.

Marina, M., \& Das, S. (2001). On-demand multipath distance vector routing in Ad Hoc Networks, in proceedings of the International Conference for Network Protocols (ICNP), 2001.

Perkins, C., \& Royer, E. (1999). Ad-Hoc on demand distance vector routing, in Proceedings of the Second IEEE Workshop on Mobile Computing Systems and Applications, Feb, pp. 90-100.

Souihli, O., Frikha, M., \& Ben-Hamouda, M. (2009). Load-balancing in MANET shortest-path routing protocols, Ad Hoc Networks, 431- 442. http://dx.doi.org/10.1016/j.adhoc.2008.04.007

Tran, A., \& Raghavendra, H. (2006). Congestion adaptive routing in mobile Ad Hoc Networks. IEEE transactions on parallel and distributed systems; November, 17(11), 294-1305.

Tsai, J., \& Moors, T. (2005). A review of multipath routing protocols: From wireless Ad Hoc to Mesh Networks. Communications Magazine, IEEE.

Ye, Z., Krishnamurthy, S., \& Tripathi, S. (2003). A framework for reliable routing in mobile Ad Hoc Networks. in proceedings of the Twenty-Second Annual Joint Conference of the IEEE Computer and Communications Societies IEEE (INFOCOM 2003), vol. 1, pp. 270-280.

Ye, Z., Krishnamurthy, S., \& Tripathi, S. (2004). Use of congestion-aware routing to spatially separate TCP connections in wireless Ad Hoc Networks. Mobile Ad-hoc and Sensor Systems.

Zhou, A., \& Hassanein, H. (2001). Load-balanced wireless Ad hoc Routing. in proceedings of the 4th ACM international workshop on Modeling, analysis and simulation of wireless and mobile systems. 